\title{
Effect of intravitreal Ziv-aflibercept on choroidal neovascularization membrane secondary to post traumatic choroidal rupture
}

\begin{abstract}
Purpose: To report the efficacy and safety of intravitreal ziv-aflibercept in a young female with choroidal neovascularization membrane secondary to post traumatic choroidal rupture.

Methods: This was interventional case report, of a symptomatic 15 years' old female with history of blunt trauma presented for reduced vision, underwent for clinical examination and fluorescein angiography at baseline and 12 weeks of treatment with three consecutive intravitreal injection of ziv-aflibercept $1.25 \mathrm{mg}$ every 4 weeks with evaluation of ocular and systemic complications.
\end{abstract}

Results: Improvement of best corrected visual acuity from baseline LogMAR 0.30 and 0.10 at 12 weeks with marked regression of choroidal neovascularization membrane on fluorescein angiogram a 12 weeks from baseline with no signs of ocular nor systemic complications.

Conclusions: Ziv-Aflibercept seems to be safe and effective in treatment of choroidal neovascularization membrane post traumatic choroidal rupture for 12 weeks follow up.

Keywords: CNVM, anti VEGF, VEGF trap, trauma, choroidal rupture, ZALTRAP, Zivaflibercept
Volume 6 Issue 5 - 2017

\author{
Ameen Marashi \\ Marashi Eye Clinic,Aleppo, Syria
}

Correspondence: Ameen Marashi, Retina specialist at Marashi Eye Clinic, Aleppo Syria,

Email ameenmaharshi@hotmail.com, http://www.amretina.tk/

Received: April 05, 2017| Published: April 19, 2017

\begin{abstract}
Abbreviations: CNVM, choroidal neovascularization membrane; VEGF, vascular endothelial growth factor; FDA, food and drug administration
\end{abstract}

\section{Introduction}

When blunt trauma causes anterior posterior globe compression and then expansion of the globe in horizontal manner, a tear of the inner choroid along with Bruch's membrane and the RPE will form due to inflexible nature of these tissues in contrast to sclera and retina that they don't frequently rupture in blunt trauma. Choroidal ruptures can have crescent shape and can be isolated or multiple, they are usually located at temporal to the optic disc and often involve the macula which may reduce vision if fovea is involved or Asymptomatic if fovea is speared, it can be accompanied or masked with sub-retinal, retina, pre-retinal hemorrhage, or commotio retinae. Choroidal ruptures can be complicated with CNVM in 5-10\% of cases, especially in cases of long choroidal ruptures and ruptures that involve the macula, which may form more scar tissue, and reduce vision, CNVM due to choroidal ruptures may be treated with intravitreal Anti-VEGF, photo dynamic therapy (PDT) or laser photocoagulation. Several case reports (1) (2) showed that intravitreal anti-VEGF can be effective in regression of CNVM secondary to post traumatic choroidal rupture, in this case report, intravitreal $1.25 \mathrm{mg}$ Ziv-Aflibercept (ZALTRAP, SanofiAventis US, LLC, Bridgewater, New Jersey, USA and Regeneron Pharmaceuticals, Inc, Tarrytown, New York, USA) was used to treat a 15 years old female suffered from CNVM after she was exposed to ocular blunt trauma.

\section{Case report}

A 15 years old female presented to Marashi Eye Clinic because she had reduced vision in her right eye after blunt ocular trauma, her vision was $0.30 \operatorname{LogMAR}$ at presentation in her right eye where he left eye he vision was $0.00 \operatorname{LogMAR}$, subjective refraction was plano in both eyes, her medical and familial history is unremarkable. Her anterior chamber examination was unremarkable, with no signs of traumatic cataract or iris defects in both eyes, where her dilated fundus exam shows crescent shape choroidal rupture temporal to the macular between the arcades with inferior temporal retinal hemorrhage, the left eye fundus examination was normal. Fluorescein angiography was done and revealed the presence of macular active CNVM in the choroidal rupture temporal to the fovea which is presented as hyperfluoresceinced crescent shape lesion along of retinal hemorrhage which masks the temporal side of the angiogram (Figure 1). A discussion was made with girl's parents and they agreed to monthly injection of intravitreal ziv-aflibercept for 3 consecutive injections under sedation, and informed consent was obtained from the parents. Ziv-aflibercept intravitreal Injection was administered under sterile condition every 4 weeks for 3 three consecutive injections, injection site was prepared by disinfecting the skin using povidone iodine $10 \%$ and the conjunctiva using povidone iodine $4 \%$ under sedation, the injection is carried out after placing sterile drape and lid speculum isolating eye lashes in the inferior temporal quadrant, injection site was measured with calipers $4 \mathrm{~mm}$ from the limbus, 30 gauge half inch needle is used to inject $0,05 \mathrm{ml} / 1.25 \mathrm{mg}$ of Ziv-aflibercept (ZALTRAP) then cotton tip applicator is placed over the injection site 
to prevent reflux of fluid, topical and systemic antibiotics were used post injection. Best corrected visual acuity was measured at baseline, 4 weeks, 8 weeks, 12 weeks using snellen chart then converted to LogMAR, to monitor efficacy where fluorescein angiography was obtained at baseline and week 12 to evaluate CNVM activity, intraocular pressure was monitored at base line, 4 weeks, 8 weeks, and 12 weeks. Safety parameters include the presence of ocular and non-ocular side effects such as intraocular inflammation, toxicity or retinal detachment along with systemic events. After 12 weeks follow up there was no change in IOP (intraocular pressure) from baseline and where best corrected visual acuity was converted from Snellen to LogMAR, The LogMAR improved from 0.30 (20/40) at baseline to $0.20(20 / 25)$ at 12 weeks follow up, where fluorescein angiogram shows CNVM regression from baseline to 12 weeks follow up leaving scar (Figure 2). There was no evidence of intraocular inflammation nor toxicity or cataract progression neither systemic complication due to intravitreal injection of Ziv-Aflibercept.

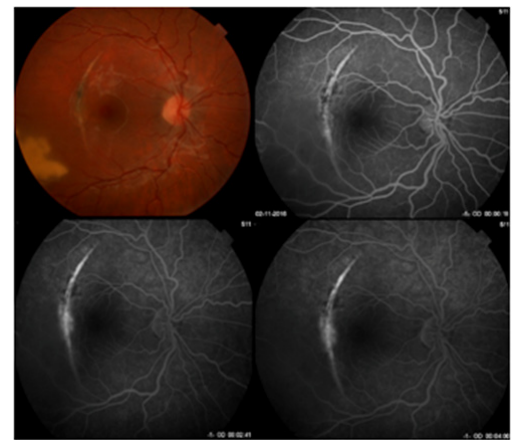

Figure I Patients fundus photograph and Fluorescein angiography at baseline shows crescent choroidal rupture with active CNVM and retinal hemorrhage temporal to the fovea.

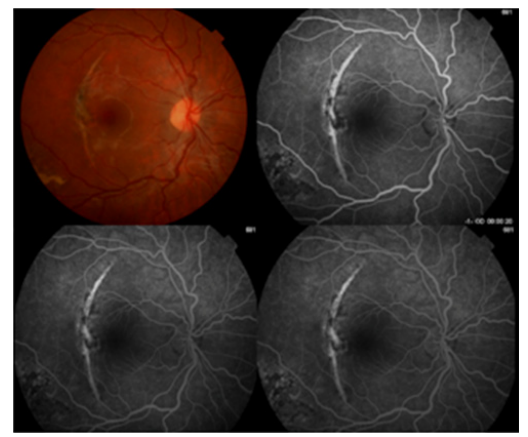

Figure 2 Patients fundus photograph and Fluorescein angiography at 12 weeks follow up after 3 consecutive intravitreal ziv-aflibercept shows crescent choroidal rupture with regressed CNVM and scar formation.

\section{Discussion}

Choroidal rupture can cause break in Bruch's membrane and the RPE which will induce inflammatory process that evoke VEGF secretion and thus will lead to CNVM progress, which interim may cause subretinal fluid or/and hemorrhage accumulation and thus will cause visual loss. Therapeutic options for CNVM secondary to choroidal rupture are observation, intravitreal Anti-VEGF, PDT or laser photocoagulation, we choose intravitreal anti-VEGF to treat the CNVM because of vision reduction (0.30LogMAR) to avoid poor visual prognosis in a young girl, along reports ${ }^{1,2}$ have shown that antiVEGF can successfully treat CNVM secondary to choroidal rupture. Ziv-aflibercept has the same fusion protein as aflibercept (Eylea, Regeneron, Tarrytown, NY) but with different buffer hyper osmolar solution $(1000 \mathrm{mOsm} / \mathrm{kg})$ which cost only about $50 \$$ for a single dose, but brought some concerns about it safety. Clinical trial conducted by Malik et al. ${ }^{3}$ to address the safety issue of ziv-aflibercept in vivo and in vitro and concluded that there is non-significant RPE toxicity where, Mansour et al. ${ }^{4}$ has studied the safety of intravitreal Ziv-aflibercept in six patients and all patients, and the results didn't show any signs of retinal, ocular toxicity or inflammation and suggested that the $0.05 \mathrm{ml} \mathrm{Ziv-aflibercept} \mathrm{is} \mathrm{diluted} \mathrm{in} 4 \mathrm{ml}$ of vitreous when intravitreally injected, thus it is 80 times diluted which means the final osmolarity of $312 \mathrm{mOsm} / \mathrm{kg}$ after intravitreal injection of $0.05 \mathrm{ml}$ Ziv-aflibercept, Masnour et al. ${ }^{5}$ also studied the safety and efficacy of intravitreal zivaflibercept in wet age related macular degeneration and concluded that intravitreal ziv-aflibercept can be safe and effective without causing any ocular toxicity.

\section{Conclusion}

This interventional case report has shown that intravitreal injection of $1.25 \mathrm{mg}$ Ziv-aflibercept for cnvm secondary to choroidal rupture as a monotherapy can improve visual acuity and induce CNVM regression within 12 weeks with no sign of retinal or ocular toxicity, However, a long term studies are required to evaluate the long term safety and effectiveness of intravitreal Ziv-aflibercept for CNVM secondary to choroidal rupture.

\section{Acknowledgments}

None.

\section{Conflicts of interest}

The authors declare there are no conflicts of interest.

\section{References}

1. De Benedetto U, Battaglia Parodi M, Knutsson KA, et al. Intravitreal Bevacizumab for Extrafoveal Choroidal Neovascularization After Ocular Trauma. J Ocul Pharmacol Ther. 2012;28(5):550-552.

2. Kim M, Kim JH, Seo Y, et al. Intravitreal Bevacizumab for Traumatic Choroidal Rupture. Optom Vis Sci. 2015;92(10): e363-e367.

3. Malik D, Tarek M, Caceres del Carpio J, et al. Safety profiles of antiVEGF drugs: bevacizumab, ranibizumab, aflibercept and ziv-aflibercept on human retinal pigment epithelium cells in culture. $\mathrm{Br} J$ Ophthalmol. 2014;98 Suppl 1: i11-16.

4. Mansour AM, Al-Ghadban SI, Yunis MH, et al. Ziv-aflibercept in macular disease. Br J Ophthalmol. 2015;99(8):1055-1059.

5. Mansour AM, Chhablani J, Antonios RS, et al. Three-month outcome of ziv-aflibercept for exudative age-related macular degeneration. $\mathrm{Br} J$ Ophthalmol. 2016;100(12):1629-1633. 